\title{
Air embolism during air flight in a passenger with bronchogenic cyst
}

\author{
Mahwish Khawar, MBBCH, ${ }^{\mathrm{a}}$ Ahmed Elmahrouk, MD, ${ }^{\mathrm{b}}$ and Amer Chaikhouni, MD, ${ }^{\mathrm{c}}$ \\ Doha, Qatar, and Tanta, Egypt
}

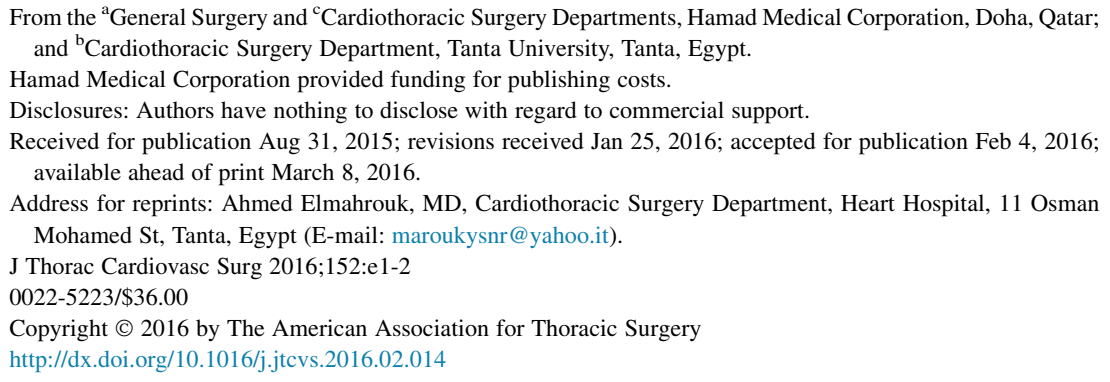

A 35-year-old man had a sudden episode of seizures and loss of consciousness on an international flight. He developed these symptoms 20 minutes after takeoff, leading to an emergency landing, and was taken to a hospital. On arrival at the emergency room, his Glasgow Coma Score was 15 (normal >14), with stable vital signs, but he had right-side hemiparesis. He maintained oxygen saturations of $98 \%$ on room air, and did not require intubation. His electrocardiogram was normal, and laboratory findings were unremarkable. He did not develop any more seizures.

This patient had a history that included a similar episode of seizures during flight, with no loss of consciousness; he continued that journey smoothly, but he was not medically investigated. For the later occurrence, a chest radiograph showed a large air-filled cyst compressing the left lung (Figure 1). A chest computed tomography (CT) scan with contrast revealed a left giant cyst measuring $9 \times 11 \times 11.3 \mathrm{~cm}$ with air-fluid level. No evidence was found of pneumothorax or pneumomediastinum. However, a mild shift of the mediastinum toward the right side was observed (Figure 2). A brain CT scan with contrast study and brain magnetic resonance imaging study showed no abnormal focal findings.

One week after admission and neurologic stabilization, he underwent elective left posterolateral thoracotomy. A tense, giant, thick-walled cyst was identified, in the posterior segment of the left upper lobe, compressing the left lung. It contained a minimal amount of blood. Marked bubbling occurred on inspiration, and a single communication was noted with the bronchial tree, near the hilar area of the cyst, measuring approximately $3 \mathrm{~mm}$. Dark blood clots found in the cyst were apparently due to small communications with pulmonary vessels. The cyst wall was approximately $2 \mathrm{~mm}$ thick and appeared smooth. The communications with the bronchial tree and pulmonary veins were sutured, and the cyst was stapled and excised. A histopathology report showed the cyst to be of bronchogenic origin (Figure 3). The patient had a

\section{DISCUSSION}

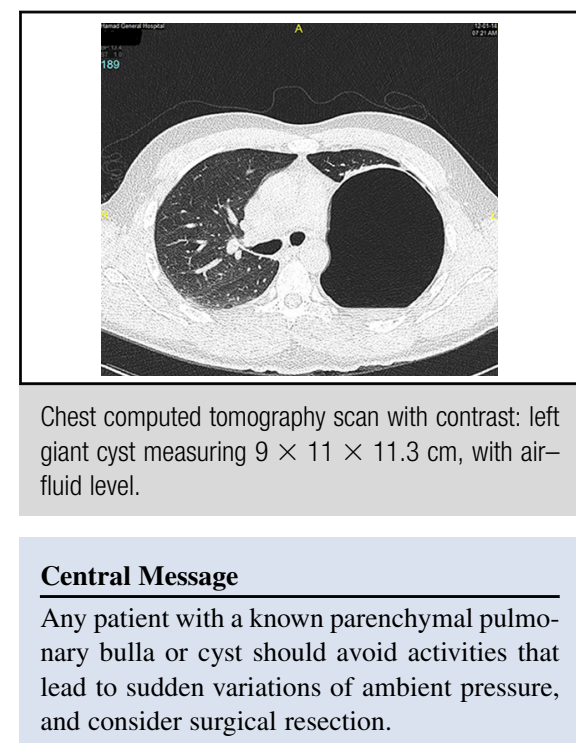

See Editorial Commentary page e3.

smooth postoperative course, with fully inflated lungs on chest radiograph. He was discharged after 7 days and cleared to fly home.

Bronchogenic cysts are relatively rare, with a prevalence of 1 in every 42,000 to 68,000 people. ${ }^{1}$ Many medical conditions are aggravated by the cabin environment in airplanes. Yet, the incidence of in-flight medical emergencies reported to the International Air Transport Association is low (16 medical emergencies per million passengers) and usually is due to cardiac-related events. ${ }^{2}$ Suspected strokes account for approximately $2 \%$, and seizures account for $5.8 \%$. Aircraft diversions are initiated by a seizure event in $12 \%$ of cases; most of these events occur in patients who have been previously diagnosed with epilepsy. ${ }^{2}$

We can find no reports describing the incidence of seizures during air travel in the presence of bronchogenic cysts. But some fatal strokes have been reported and attributed to air embolism in airline passengers who have pulmonary bronchogenic cysts. ${ }^{3}$ Our report may represent a rare case of survival with an aviation-related air embolism due to a bronchogenic cyst. A previous report indicated survival of an aircraft passenger who had a pulmonary cyst treated with repeated sessions of hyperbaric oxygen and cyst excision. Unfortunately, the pathology of the cyst was not reported. ${ }^{4}$ The authors recommended that any patient with a 


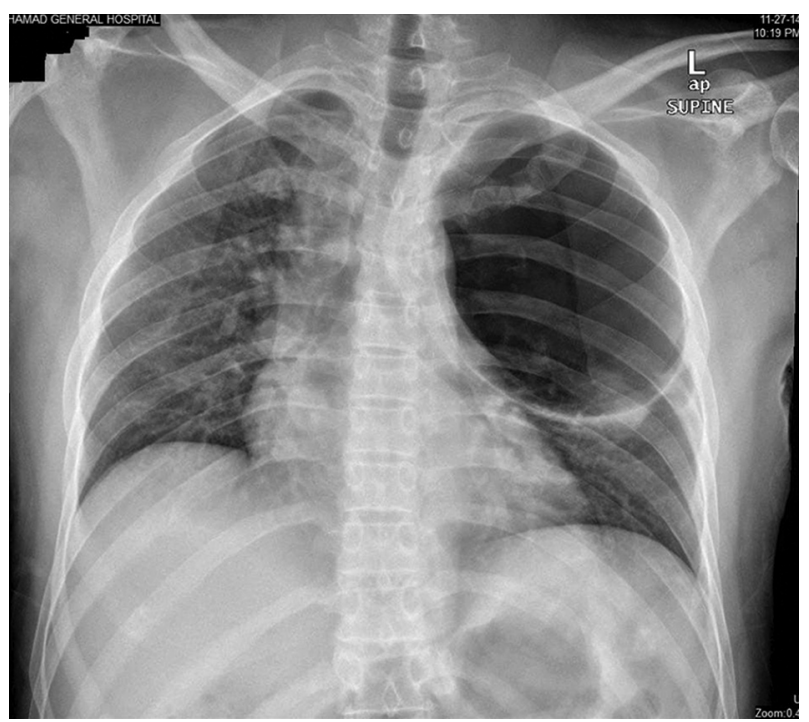

FIGURE 1. Chest radiograph showing large, air-filled cyst or giant bulla compressing the left lung.

known parenchymal pulmonary bulla should avoid activities leading to sudden variations of ambient pressure.

Aircraft regulations require that the air pressure inside the cabin not fall below the equivalent of the pressure at an altitude of 8000 feet in normal operations. As the aircraft climbs, the pressure inside the cabin decreases, relative to sea level, and gases expand in accordance with Boyle's Law (pressure $\times$ volume $=$ constant). At a cabin altitude of 8000 feet, the volume of a gas will have expanded by

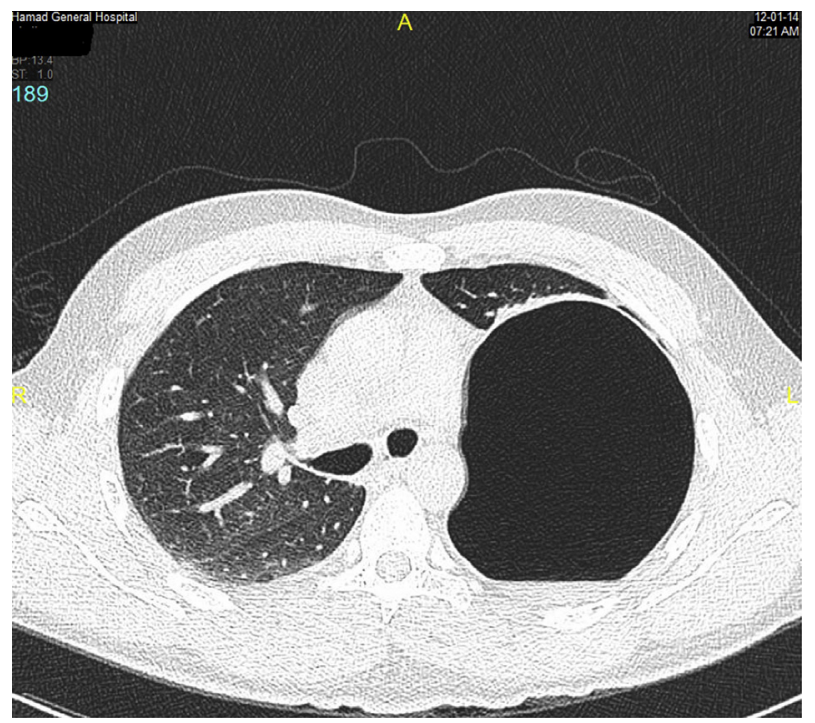

FIGURE 2. Chest computed tomography scan with contrast showing left giant cyst measuring $9 \times 11 \times 11.3 \mathrm{~cm}$, with air-fluid level, and mild shift of mediastinum to the right. No pneumothorax or pneumomediastinum is present.

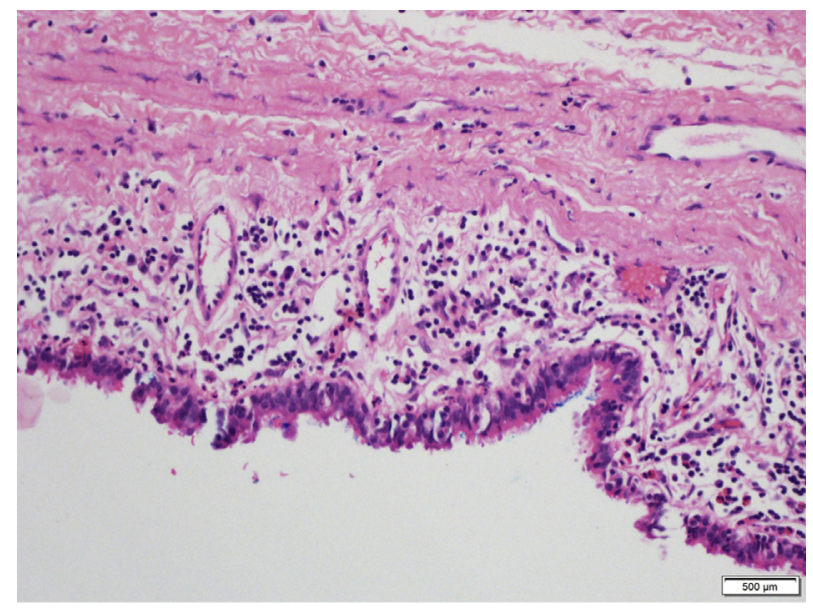

FIGURE 3. Histopathology slide of the cyst wall clearly shows the respiratory epithelial lining.

approximately $30 \% .^{5}$ These changes in gas volumes do not cause any problems where gas movement can take place freely, such as in the airways, but may cause discomfort or even tissue injury where gas is trapped or restricted. ${ }^{5}$ In a thick-walled, poorly compliant cyst, an increase in gas volume may cause an increase in pressure. Expansion of the cyst may cause small injuries to its wall and allow entry of air into the vascular system and development of air embolism.

Although magnetic resonance imaging showed the brain to be normal, the diagnosis of air embolism was based on the clinical picture, and the quick recovery of the neurologic symptoms. This patient experienced a similar episode of seizure during a flight a few years before, with no loss of consciousness. He did not fly on an aircraft between the 2 episodes, and he never experienced any neurologic symptoms except on these 2 occasions. Given that other neurologic, psychological, and general medical causes of seizures were ruled out during this admission, the presence of a large bronchogenic cyst was correlated with the neurologic event.

\section{References}

1. Coselli M, de Ipolyi P, Bloss RS, Diaz RF, Fitzgerald JB. Bronchogenic cysts above and below the diaphragm: report of eight cases. Ann Thorac Surg. 1987; 44:491-4.

2. Cummins RO, Chapman PJC, Chamberlain DA, Schubach JA, Litwin PE. In-flight deaths during commercial air travel: How big is the problem? JAMA. 1988;259: 1983-8.

3. Edwardson M, Wurth D, Lacy JM, Fink J, Becker K. Cerebral air embolism resulting in fatal stroke in an airplane passenger with a pulmonary bronchogenic cyst. Neurocrit Care. 2009;10:218-21.

4. Closon M, Vivier E, Breynaert C, Duperret S, Branche P, Coulon A, et al. Air embolism during an aircraft flight in a passenger with a pulmonary cyst: a favorable outcome with hyperbaric therapy. Anesthesiology. 2004;101:539-42.

5. British Thoracic Society Standards of Care Committee. Managing passengers with respiratory disease planning air travel: British Thoracic Society recommendations. Thorax. 2002;57:289-304. 